\section{In vivo gated 4D imaging of the embryonic heart using optical coherence tomography}

\author{
Michael W. Jenkins, ${ }^{\mathrm{a}, *}$ Osman Q. Chughtai, ${ }^{\mathrm{a}}$ \\ Ajay N. Basavanhally, ${ }^{a}$ Michiko Watanabe, ${ }^{b}$ and \\ Andrew M. Rollins ${ }^{\mathrm{a}}$ \\ ${ }^{\mathrm{a} C}$ ase Western Reserve University, Department of \\ Biomedical Engineering, 11100 Euclid Avenue, Cleveland, \\ Ohio 44106 \\ ${ }^{\mathrm{b}}$ Case Western Reserve University School of Medicine, \\ Department of Pediatrics, 11100 Euclid Aveune, Cleveland, \\ Ohio 44106
}

\begin{abstract}
We demonstrate the first in vivo gated 4D images of avian embryonic hearts by use of optical coherence tomography (OCT). We present a gated 4D dataset of an in vivo beating quail heart consisting of $\sim 864,000$ $A$-scans accumulated over multiple heartbeats. Generation of a gating trigger from a laser Doppler velocimetry (LDV) signal, collected from an outlying vitelline vessel, enabled us to gate image acquisition to the cardiac cycle. To fully characterize the genesis and mechanisms of cardiac defects, a tool capable of assessing structure and function simultaneously at early stages of development is needed, and gated OCT has the capability to become such a tool. () 2007 Society of Photo-Optical Instrumentation Engineers. [DOI: 10.1117/1.2747208]
\end{abstract}

Keywords: optical coherence tomography; embryonic hearts; medical imaging; avian embryos; gated imaging.

Paper 06333LRR received Nov. 14, 2006; revised manuscript received Apr. 13, 2007; accepted for publication Apr. 17, 2007; published online Jun. 12, 2007.

\section{Introduction}

The developing heart is a challenging subject to image in vivo because it is tiny, beating, and rapidly undergoing complex morphogenesis in three dimensions. Gated cardiac imaging (acquiring image data synchronized to the heart cycle) mitigates motion artifact due to the beating heart. Gated cardiac imaging systems can acquire 4D image sets of the heart built up over many heart cycles, which can then be used to calculate various anatomical and physiological parameters (e.g., wall thickness and ejection fraction). Preseptated embryonic avian and murine hearts (which are less than $2 \mathrm{~mm}$ in diameter) are common models for studying the etiology of congenital heart defects in the early stages of development. Confocal microscopy, which has retrospectively synchronized nongated slices in embryonic zebrafish hearts, ${ }^{1}$ lacks the field of view and depth range for imaging the avian embryo in vivo. Therefore, an imaging tool with adequate resolution and field of view capable of assessing structure and function of the embryonic heart simultaneously at early stages of development is needed.
Optical coherence tomography (OCT) $)^{2}$ measures coherently gated back-reflected light with 2 - to $15-\mu \mathrm{m}$ resolution to a depth of 1 to $2 \mathrm{~mm}$ in cardiac tissue. The high resolution and appropriate field of view make OCT a worthy technology to adapt to the study of cardiac development. This potential has been established in demonstration studies using several animal models. ${ }^{3-9}$ Recently, Yelin et al. ${ }^{4}$ imaged the embryonic Xenopus-laevis heart with high-speed (4D at $54 \mathrm{kHz}$ $A$-line rates) and high-resolution (2-D+time) OCT systems. We have demonstrated gated 4D imaging of the paced, excised embryonic chick heart and murine heart by OCT. ${ }^{8}$

The goal of this work is to demonstrate the capability of OCT to achieve gated 4D imaging of early (preseptated) avian embryo hearts in vivo with full spatial resolution. Other medical imaging modalities derive a cardiac gating trigger from the electrocardiogram of the patient. Because the electrical signal from a preseptated avian heart is minute, electrodes would have to be placed against the heart, which is invasive from the perspective of the embryo. The exact placement of the electrodes is extremely challenging and it is questionable whether adequate signals can be obtained from early embryos. In this work, we demonstrate cardiac gated OCT using a trigger derived from a laser Doppler velocimetry (LDV) signal, which is noninvasive and easily repeatable. This gated 4D image set of a stage 14 quail embryo in vivo reveals the future potential of OCT as an imaging tool capable of longitudinally assaying cardiac structure and function simultaneously, which will be an invaluable tool for characterizing the genesis and mechanisms of cardiac defects.

\section{Experimental Methods}

Avian embryos were chosen for study because their hearts develop similarly to that of humans and serve as a good animal model system, and they are easily manipulated. Fertilized quail eggs were incubated in a humidified, forced draft incubator (GQF Manufacturing, Savannah, Georgia) at $39^{\circ} \mathrm{C}$. After $48 \mathrm{~h}$, the eggs were removed from the incubator, cracked, and placed in a 3.6-cm-diam Petri dish. Quail were chosen because they are commonly used to investigate developmental mechanisms of normal and abnormal cardiac embryogenesis and the role they play in the formation of congenital heart defects. Also, the quail, while very similar to the chicken (a more commonly used model) in cardiac development, is slightly smaller than the chicken and easier to culture ex ovo at early stages of development.

The dish containing the cultured stage 14 embryo was positioned on a temperature-controlled heating pad $\left(37^{\circ} \mathrm{C}\right)$ under the OCT scanner. At this stage of development, the heart is tubular in form and has begun looping, which will ultimately lead to septation and the development of a fourchambered heart. The OCT imaging system consisted of a time-domain OCT engine (4000 A-lines per second $)^{10}$ and a microscope integrated scanner. ${ }^{11}$ The axial and lateral resolution of the system was approximately $10 \mu \mathrm{m}$ in tissue.

A commercial LDV needle probe (Moor Instruments Incorporated, Wilmington, Delaware) was positioned at $45 \mathrm{deg}$ to the sample adjacent to one of the vitelline vessels using a micromanipulator. An example of the LDV signal is shown in Fig. 1. The LDV signal was amplified (200 to 300\%) and 


\section{JBO LETTERS}

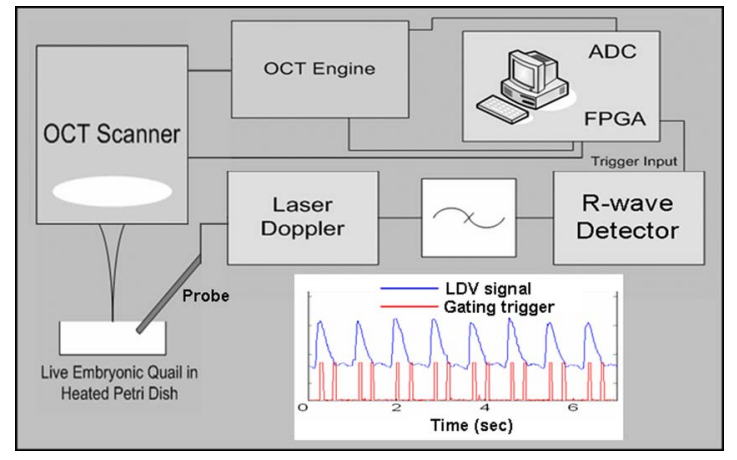

Fig. 1 Gated OCT instrumentation and signals. The LDV output was filtered and input into an R-wave detector. The R-wave detector produced two triggers per heartbeat, the first at the peak of the LDV waveform and another at the bottom of the downward slope. The rising edge of the first trigger from each heartbeat activated an FPGA board, while the FPGA was programmed to ignore the second trigger. The FPGA controls scanning and data acquisition. The inset graph shows the LDV signal (top trace) and the gating trigger output of the $\mathrm{R}$-wave detector (bottom trace). FPGA is the field programmable gate array, ADC is the analog to digital converter, and LDV stands for laser Doppler velocimetry.

low-pass filtered (Butterworth, 5-Hz corner frequency). The output of the filter was input to a commercial R-wave detector (AccuSync Medical Research Corporation). The R-wave detector produced two triggers per heartbeat, the first at the peak of the LDV waveform and another at the bottom of the downward slope (Fig. 1). The rising edge of the first trigger from each heartbeat activated a field-programmable gate array (FPGA) board, while the FPGA was programmed to ignore the second trigger. For each heartbeat, the FPGA used the line sync signal from the OCT delay line to generate $x-y$ scanning and frame sync signals, which enabled the collection of several $B$-scans (eight phases presented here) at a single location. The $B$-scans recorded during four sequential heart beats were averaged to reduce speckle noise before translating the scan beam to the next transverse location. Gated OCT allows one to average images over many heart cycles without sacrificing spatial or temporal resolution, as long as the heart is beating stably. The triggering and scanning parameters could be adjusted to accommodate a range of heart rates (around 1 to $4 \mathrm{~Hz}$ ). Figure 1 shows a diagram of the gating instrumentation and signals.

The gated 4D dataset was visualized using Analyze 6.0 (Mayo Clinic, Rochester, Minnesota) and Matlab 7.0 (Mathworks, Incorporated, Natick, Massachusetts). Four images were averaged at each position and heart-cycle phase to reduce speckle noise, which improves subsequent visualization and segmentation. The aspect ratio was corrected by considering the index of refraction of the tissue and the spatial sampling rate in each direction. Manually segmenting the heart tube from surrounding tissue in the 2D images facilitated 3D surface rendering of the heart (Fig. 2). Sum voxel projection requires no segmentation and enables evaluating of the inner structure of the heart (Fig. 3).

\section{Results}

The gated 4D image data presented here, recorded from a stage 14 quail embryo heart, consists of $\sim 864,000 \mathrm{~A}$-lines and represents $3 \mathrm{D}$ volumetric images at eight phases in the cardiac cycle. The imaging region was $1 \mathrm{~mm}$ (300 pixels) by $0.45 \mathrm{~mm}$ (90 pixels) by $1.6 \mathrm{~mm}$ (550 pixels) in depth. The duty cycle of our system is approximately $75 \%$, as each sequential $B$-scan is separated by $95 \mathrm{~ms}$, but is recorded in $71 \mathrm{~ms}$. The total imaging time was $\sim 7 \mathrm{~min}$. An average correlation coefficient of 0.9 was calculated between frames being averaged, which confirms stable gated imaging capable of making accurate physiological measurements. Although the embryo was positioned on a heating pad during imaging, the heart rate was slightly depressed ( 75 beats per minute).

Figure 2 displays representative image slices from the 4D dataset. In each slice orientation, one can readily observe the heart transition from systole in phase 1 to diastole in phase 8 . Phase 8 and phase 1 volumes were used to measure an ejection fraction of $68 \%$ with techniques described in Ref. 8 .

Figure 3 compares coronal OCT slices in diastole and systole with sum voxel projection images in diastole and systole. The OCT slices clearly resolve important anatomical features such as the compact myocardium and the endocardium, and
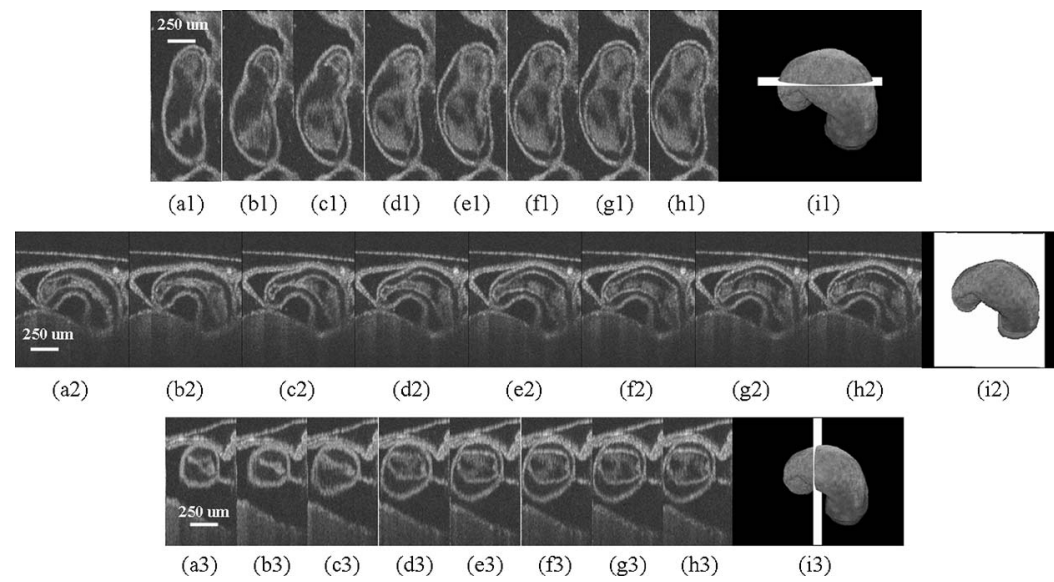

(i2)

Fig. 2 Eight phases of the beating embryonic quail heart from three different orientations acquired in vivo using LDV gated OCT. Each time series progresses from systole to diastole and each slice is separated by $95 \mathrm{~ms}$. Row 1 presents en face 2D OCT images (saggital to the body). Row 2 displays eight phases from the normal OCT view (coronal to the body), while row 3 shows the transverse view of the heart. Images on the far right show a 3D surface reconstruction of the heart in phase 8 (diastole). The white plane indicates the location of the preceding $2 \mathrm{D}$ OCT images. 


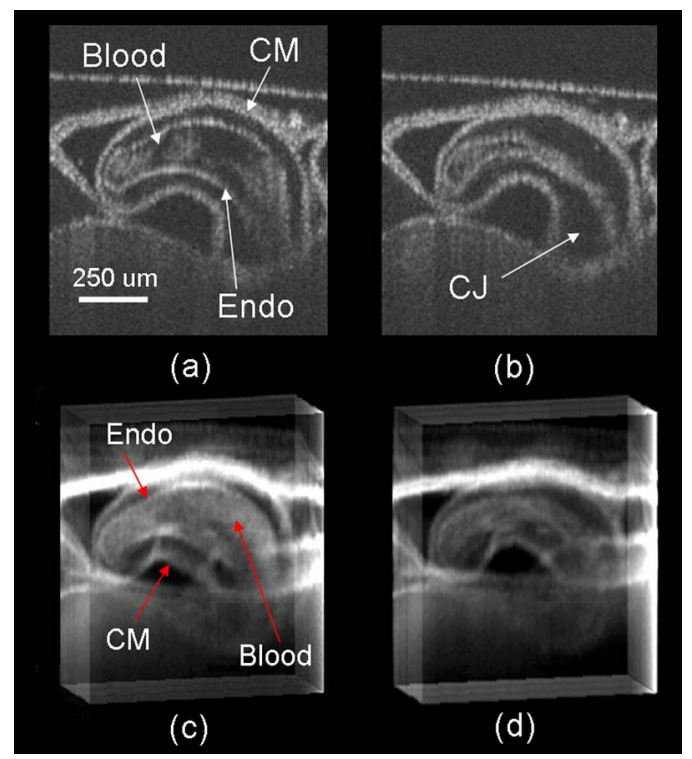

Fig. 3 LDV gated OCT images (systole versus diastole). (a) 2D OCT image in diastole cut coronal to the body of the embryo. (b) 2D OCT image in systole cut coronal to the body of the embryo. (c) Sum voxel projection image in diastole. (d) Sum voxel projection image in systole. Sum voxel images projected signal intensity along the sagittal orientation. The OCT images clearly show the compact myocardial and endocardial borders of the heart and demonstrate the quality capable with this technique, while sum voxel projection allows us to interpret a whole 3D dataset, where both the outer and inner surfaces are visible. CM is the compact myocardium, endo is the endocardium, and $\mathrm{C}$ is the cardiac jelly.

demonstrate the image quality achievable with this technique. Sum voxel projection allows us to easily visualize and interpret a 3D image, revealing both the outer and inner surfaces of the heart and how the heart is positioned within the surrounding tissue. The 3D structure of the heart becomes further evident by projecting along various angles and assembling a rotational movie (not shown).

\section{Discussion and Conclusions}

We present a gated 4D dataset of an in vivo beating quail heart consisting of $\sim 864,000 A$-scans accumulated over multiple heartbeats. To capture a $1 \times 1-\mathrm{mm}$ region sampling spatially at the Nyquist rate at 30 volumes per second, one would need an OCT system capable of imaging 1,200,000 scans per second (averaging not included). Unfortunately, the fastest system to date, which incorporates a Fourier Domaine Mode Locked (FDML) tunable laser, operates at $370 \mathrm{kHz} .{ }^{12}$ Ideally, a gated system integrated with a fast tunable laser would give better results. This would allow high enough temporal resolution to capture the dynamics of the heart wall motion in systole, which would require approximately 100 phases in the heart cycle.

Gated 4D OCT has some limitations and some advantages when compared to 4D imaging without gating. Gated 4D imaging is unable to visualize or track blood cells flowing, imaging time is much longer, and abnormal beats or drifting during imaging could cause a loss of resolution. Some of the advantages of gated imaging include the ability to observe larger areas, average frames without a loss in resolution, and increase temporal resolution for observing systolic dynamics.

Gated OCT offers the ability to assess structure and function simultaneously, and our demonstration points to the vast potential of the technique. In the future, strategies to control physiological conditions of the embryo will enable reliable longitudinal studies. In vivo gated OCT imaging of avian embryo hearts will allow cardiac researchers to investigate aspects of normal development that were until now inaccessible for analysis.

\section{Acknowledgments}

The authors acknowledge support from the Research Facilities Improvement Program C06 RR1246-01 from the National Center for Research Resources (NIH). Also, support for the work came from grant number 1RO1HL08304 (NIH) and a Case Western Reserve University startup grant.

\section{References}

1. M. Leibling, A. S. Forouhar, M. Gharib, S. E. Fraser, and M. E. Dickinson, "Four-dimensional cardiac imaging in living embryos via postacquisition synchronization of nongated slice sequences," $J$. Biomed. Opt. 10(5), 050401 (2005).

2. D. Huang, E. A. Swanson, C. P. Lin, J. S. Schuman, W. G. Stinson, W. Chang, M. R. Hee, T. Flotte, K. Gregory, C. A. Puliafito, and J. G. Fujimoto, "Optical coherence tomography," Science 254, 1178-1181 (1991).

3. S. A. Boppart, G. J. Tearney, B. E. Bouma, J. F. Southern, M. E. Brezinski, and J. G. Fujimoto, "Noninvasive assessment of the developing Xenopus cardiovascular system using optical coherence tomograpy," Proc. Natl. Acad. Sci. U.S.A. 94, 4256-4261 (1997).

4. R. Yelin, B. E. Bouma, D. Yelin, S. A. Yun, C. Boudoux, W. Y. Oh, B. J. Vakoc, and G. J. Tearney, "High-speed and high-resolution optical imaging of the developing Xenopus laevis myocardium," Biomed. Opt. (BiOS) (2006).

5. T. M. Yelbuz, M. A. Choma, L. Thrane, M. L. Kirby, and J. A. Izatt, "Optical coherence tomography a new high-resolution imaging technology to study cardiac development in chick embryos," Circulation 106, 2771-2774 (2002).

6. A. M. Davis, F. G. Rothenberg, and J. A. Izatt, "Volumetric imaging of chick embryo heart development in vivo using a high speed Doppler spectral domain OCT microscope," Proc. SPIE 6429, 64290R (2002).

7. W. Luo, D. L. Marks, T. S. Ralston, and S. A. Boppart, "Threedimensional optical coherence tomography of the embryonic murine cardiovascular system," J. Biomed. Opt. 11(2), 021014 (2006).

8. M. W. Jenkins, F. Rothenberg, D. Roy, Z. Hu, V. P. Nikolski, M. Watanabe, D. L. Wilson, I. R. Efimov, and A. M. Rollins, "4D embryonic cardiography using gated optical coherence tomography," Opt. Express 14, 736-748 (2006).

9. M. W. Jenkins, P. Patel, H. Deng, M. M. Montano, M. Watanabe, and A. M. Rollins, "Phenotyping transgenic embryonic murine hearts using optical coherence tomography," Appl. Opt. 46, 1776-1781 (2006).

10. A. M. Rollins, S. Yazdanfar, M. Kulkarni, R. Ung-Arunyawee, and J. Izatt, "In vivo video rate optical coherence tomography," Opt. Express 3, 219-229 (1998).

11. Z. Hu and A. M. Rollins, "Quasi-telecentric optical design of a microscope-compatible OCT scanner," Opt. Express 13, 6407-6415 (2005).

12. R. Huber, D. C. Adler, and J. G. Fujimoto, "Buffered Fourier domain mode locking: unidirectional swept laser sources for optical coherence tomography imaging at 370,000 lines/s," Opt. Lett. 31, 29752977 (2007). 